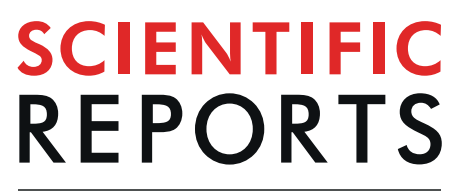

natureresearch

\title{
The role of attention in the relationship between early life stress and depression
}

\begin{abstract}
Yu Mao ${ }^{1,2,4}$, Hong Xiao ${ }^{1,2,4}$, Cody Ding ${ }^{2,3 *}$ \& Jiang Qiu ${ }^{1,2 *}$
Early life stress (ELS) can be very harmful to an individual's wellbeing and brain development. It is well established that childhood maltreatment is a significant risk factor for depression. ELS is positively correlated with depressive symptoms both in major depression disorder patients and healthy individuals, but the cognitive and neural mechanisms underlying this association are still unclear. In the present study, we calculate the within/between-network connectivity in 528 college students, and Pearson correlation was performed to investigate the relationship between network measures and ELS. Additionally, the same method was applied to verify these results in another sample. Finally, mediation analysis was performed to explore the cognitive and neural mechanisms regarding the association between ELS and depression. Correlation analysis indicated that ELS was positively correlated with the within-network connectivity of the ventral attention network (VAN), the dorsal attention network (DAN), the salience network (SN), the somatosensory network (SMN) and the between-network connectivity of ventral attention network-dorsal attention network (VAN-DAN), ventral attention network- somatosensory network (VAN-SMN), and ventral attention networkvisual network (VAN-VN). Validation results indicated that ELS is associated with the within-network connectivity of VAN and DAN. Mediation analysis revealed that attention bias and the within-network connectivity of VAN could mediated the relationship between ELS and depression. Both behavioral and neural evidence emphasize ELS's influence on individual's emotion attention. Furthermore, the present study also provides two possible mediation models to explain the potential mechanisms behind the relationship between ELS and depression.
\end{abstract}

ELS refers to traumatic life event occurring childhood, including emotional, physical, and sexual abuse, emotional and physical neglect. Evidence suggests that ELS leads to prolonged phases of stress and even predict negative outcomes over the course of a lifetime ${ }^{1}$. In particular, maltreated children are more likely to develop posttraumatic stress disorder (PTSD) $)^{2,3}$, major depression disorder $(\mathrm{MDD})^{4-6}$, anxiety disorder ${ }^{7,8}$, and substance abuse $^{9,10}$ in the future. In addition, childhood maltreatment is also associated with lower level of well-being ${ }^{11,12}$, worse educational outcomes ${ }^{13,14}$ and shortened life expectancy ${ }^{15}$. With the development of MRI technology, Empirical researches have explored the influence of ELS on individual's brain. The most prominent results oriented to the hippocampus and amygdala-brain regions which are sensitive to stress ${ }^{16-19}$. For example, adults who had experienced ELS demonstrated smaller volume of hippocampus, compared with controls ${ }^{20}$. ELS was associated with decreased amygdala volume ${ }^{21}$. Besides, studies have indicated that ELS also influence the volume of the prefrontal cortex, sensorimotor cortex and its related fiber-tract integrity ${ }^{21}$. These findings provide valuable evidences for comprehending how ELS modifies individual's brain. However, recent studies confirmed that the human brain was consisted with complicated functional networks, and cognitive and affective processing are supported by the interaction of these networks, instead of the function of a single brain area. Thus, new pointcut is needed to explore the modifications and alternations that ELS makes to brain networks.

It has been suggested that identifying and processing emotional visual information is essential for emotion regulation ${ }^{22}$ and emotional attention bias is one of the most significant cognitive features in individuals who were exposed to ELS ${ }^{23,24}$. Empirical studies have supported that individuals with ELS demonstrated attention bias either toward or away from emotional stimuli ${ }^{9,25-27}$. Attention bias arises as early as 150 milliseconds

${ }^{1}$ Key laboratory of cognition and personality (SWU), Ministry of Education, Chongqing, 400715, China. ${ }^{2}$ Department of psychology, Southwest University, Chongqing, 400715, China. ${ }^{3}$ Education science \& professional programs, University of Missouri-St. Louis, Louis, United States. ${ }^{4}$ These authors contributed equally: Yu Mao and Hong Xiao. *email:dingc@umsl.edu; qiuj318@swu.edu.cn 


\begin{tabular}{|l|l|l|l|l|l|l|}
\hline \multirow{2}{*}{ Variables } & \multicolumn{3}{|l|}{ Dataset 1 (n=528) } & \multicolumn{3}{l|}{ Dataset 2 (n=208) } \\
\cline { 2 - 7 } & Mean & SD & Range & Mean & SD & Range \\
\hline Age & 19.42 & 1.40 & $16-26$ & 22.30 & 1.47 & $18-27$ \\
\hline ELS & 37.35 & 8.34 & $25-72$ & 35.39 & 9.16 & $25-77$ \\
\hline Depression & 7.05 & 6.45 & $0-30$ & 7.24 & 5.76 & $0-27$ \\
\hline Present stress & 46.70 & 20.88 & $0-132$ & 46.43 & 31.37 & $0-131$ \\
\hline
\end{tabular}

Table 1. Demographic data. Note: $\mathrm{SD}=$ standard deviation;

after the presentation of the stimulus and modulate reactions occurring at this time scale usually depend on stimulus-driven (as opposed to voluntary) attention, a function rely on the participation of the VAN ${ }^{9,28}$. Considering the role of VAN in orienting and responding to salient stimuli ${ }^{29}$. It is reasonable to infer that attention bias might depend on the function of VAN.

ELS is believed to be a key risk factor for developing major depression disorder (MDD) ${ }^{30,31}$. Furthermore, previous studies have shown that there is a significant relationship between ELS and depression $\operatorname{mood}^{32,33}$. But the neural and cognitive mechanisms behind this association is still ambiguous. Thus, it is of great significance to discover the cognitive mechanism of how childhood maltreatment influence individual's depressive emotion. In a sense, it may help to propose relevant interventions to decrease the negative influence of ELS. Evidence demonstrated that experimentally inducing attention bias to emotionally valanced stimulus causes measurable changes in mood in healthy individuals ${ }^{34,35}$, whereas attention bias modification can alleviate depressive symptoms ${ }^{36,37}$. Attention bias to traumatic experience might be an adaptive strategy to prepare for the forthcoming threat ${ }^{38}$, but from a long-term perspective, it will interfere with subsequent information processing, which might lead to a consistent negative influence. Presumably, we can infer that individual exposed to ELS might develop attention bias to emotionally valenced, which is detrimental to effective emotion regulation, and ultimately result in high level of depression when facing with stress. Both clinical and empirical studies emphasize the role of attention bias in developing and maintaining depression ${ }^{39,40}$. Thus we hypothesized that attention bias might play a key role in the relationship between ELS and depression,

Thus, the present study was designed to explore the influence of ELS on individual's brain networks. Furthermore, we also attempted to decipher the neural and cognitive mechanism sustaining the association between ELS and depression. First, we investigate the relationship between ELS and within/between network connectivity with a big sample of 528 college students. To verify the results, machine learning method was applied to another small sample. Second, mediation analysis was performed to test whether there were particular neural basis mediate the relationship between ELS and depression. Finally, we explored whether attention bias could mediate the relationship between ELS and depression. Based on previous evidence, we hypothesized that attention bias and the functional connectivity (FC) of attention networks might be the behavioral and neural markers of ELS. In addition, there were two possible mediation mechanisms which might elucidate the potential neural and cognitive mechanisms sustaining the relationship between ELS and depression.

\section{Method}

Participants. Dataset 1 contained 528 right-handed college students ( 157 males, age $=19.42 \pm 1.40$ years, age range: 16-26 years), 38 participants were excluded for its maximal motion between volumes in each direction $>3 \mathrm{~mm}$, and rotation about each axis $>3^{\circ}$. Dataset 2 contained 208 right-handed college students (86 males, age $=22.30 \pm 1.47$ years, age range: $18-27$ years), 25 participants were excluded for its maximal motion between volumes in each direction $>3 \mathrm{~mm}$, and rotation about each axis $>3^{\circ}$ (see Table 1). Participants with a history of manic episodes, psychotic features or neurological illness were excluded. All participants were college students from Southwest University in China. They were informed of the procedure of the experiment, and finished written informed consent prior to participating in the study. For the participants under the age of 18 years, their parents were informed of the procedure and allowed them to participate in the experiment, and finished written informed consent prior to participating in the study. The study was approved by the Southwest University Brain Imaging Center Institutional Review Board. All research was performed in accordance with the relevant guidelines and regulations.

Measure of the behavior variable. The childhood trauma questionnaire. The Childhood Trauma Questionnaire-Short Form (CTQ-SF) was used to evaluate individual's traumatic experience during childhood ${ }^{41}$. The CTQ consisted of 25 clinical questions and 3 validity items. All the items were rated on a 5-point Likert scale (ranging from 1 never true to 5 very often true) enquiring about 5 types of childhood trauma including emotional, physical and sexual abuse and emotional and physical neglect. The CTQ-SF has demonstrated good reliability and validity ${ }^{42}$. The Cronbach's alpha of DAQ in the dataset 1 was 0.74 , indicating that the present questionnaire had good construct validity.

Depression. Individual's difference in depression was evaluated by Beck Depression Inventory ${ }^{43}$. The BDI-II is a 21-item self-report questionnaire which measured the severity of depressive symptoms within the past week. Each of the 21 items was rated on 4-point Likert-type scale ranging from 0-3. Participants who get higher score in the BDI tend to experienced more depressive symptoms. The BDI- II is a reliable measure which is widely used to assesses the severity of depressive symptoms from non-clinical to clinical samples ${ }^{44}$.The Cronbach's alpha of the scale was 0.85 in this sample, 
Current stress. The Current stress was accessed by the Adolescent Self-Rating Life Events Checklist ${ }^{45}$. It consisted of 27 common negative life events which belonged to multiple social-stress domains: family (e.g., "Physical punishment by parents"), school (e.g., "Failure in a test"), interpersonal (e.g., "Break up with close friends"), and personal physical diseases (see Table 1), these life events were suggested to be the most frequently occurred to Chinese adolescents ${ }^{45}$. Each item begins with whether the participants experienced the particular event, and then there is a five-point Likert scale (ranging from 1 "not at all" to 5 "extremely severe") to evaluate the perceived stress of each life event. The Cronbach's alpha of the scale was 0.95 in this sample,

Dot probe task. 102 participants completed the experiment outside of the MRI scanner. Eprime 2.0 software (https://pstnet.com/products/e-prime/) was used to control the presentation of stimuli and to record response accuracy and response time. The dot probe task was designed according to MacLeod ${ }^{46}$. At the beginning of the task, there was a fixation cross presented for $500 \mathrm{~ms}$ on the screen, followed by a pair of faces for $500 \mathrm{~ms}$, after which the dots appeared, then the dots disappeared. The participants were then asked to identify whether the small dots were arranged horizontally or vertically by pressing the associated key. Before the real experiment started, participants were given five practice trials to familiarize themselves with the procedure. The task contained 8 conditions: 4 emotion face pairs (neutral and happy, NH, neutral and sad, NS, neutral and anger, NF, neutral and fear) $\mathrm{x} 2$ probe locations (in the location of the emotional face or the neutral face) and was comprised of 2 blocks of 151 trails (NH 40 trails, NS 37 trails, NA 37 trails, NF 37 trails), with a short break between the blocks. Attention bias scores were calculated as follows:

Attentional bias score $=1 / 2[(\mathrm{RpLe}-\mathrm{RpRe})+(\mathrm{LpRe}-\mathrm{LpLe})]^{47}$

$\mathrm{R}=$ right position; $\mathrm{L}=$ left position; $\mathrm{p}=$ probe; $\mathrm{e}=$ emotional face. In this equation, RpLe represents the mean reaction time when the probe presents in the left and the emotional face presents in the right, and so on. This equation estimates the "attention capturing" quality of emotional faces by subtracting the mean reaction times for probes presenting in the same position of the emotional face from the mean reaction times for probes presenting in the opposite position of the emotional face ${ }^{47}$. Positive bias scores represent the allocation of attention toward the emotional face compare with the matched neutral faces, and negative bias scores represent the allocation of attention away from the emotional face relative to the matched neutral faces, that is attention avoidance.

RsfMRI data acquisition and preprocessing. Image acquisition. Whole-brain images were quarried by a Siemens 3.0 T magnetic resonance scanner. Participant's high-resolution anatomical image data was acquired through a T1- weighted magnetization-prepared rapid gradient-echo (3d MP-RAGE) sequence in sagittal plane (inversion time $=900 \mathrm{~ms}$, repetition time $=1900 \mathrm{~ms}$, echo time $=2.52 \mathrm{~ms}$, flip angle $=9^{\circ}$, resolution matrix $=$ $256 \times 256$, thickness $=1.0 \mathrm{~mm}$, slices $=176$, voxel size $=1 \times 1 \times 1 \mathrm{~mm}^{3}$ ). The functional image was coregistrated to $\mathrm{T} 1$ image.

The resting-state fMRI data scanning took 8 minutes, all participants were instructed to have a rest while keeping eyes closed, but stay awake during scanning. Participant's whole brain functional slices were acquired in descending order by gradient-echoplanar imaging (EPI) sequences, and the detailed parameters were as follows: repetition time $(\mathrm{TR})=2000$, echo time $(\mathrm{TE})=30 \mathrm{~ms}$, slices $=32$, flip angle $=90^{\circ}$, field of view $(\mathrm{FOV})=220 \mathrm{~mm}$ $\times 220 \mathrm{~mm}$, andvoxel size $3.4 \times 3.4 \times 4 \mathrm{~mm}^{3}$, thickness $=3 \mathrm{~mm}$, slice gap $=1 \mathrm{~mm}$.

Image data preprocessing. Imaging data was applied with SPM8 toolbox (http://www.fil.ion.ucl.ac.uk/spm/software/spm8/) and Data Processing Assistant for Resting- State fMRI (DPARSF) based on MATLAB 2012a platform (MathWorks Inc., Natick, MA) to preprocess. The first 10 EPI images were removed considering that the participants need time to get used to the scanning circumstance, and slice timing were conducted in the remaining images. Then the neuroimaging data underwent standard realignment and normalization (MNI space). Next the fMRI time-series were entered into spatial smoothing ( $8 \mathrm{~mm}$ FWHM). Additionally, band-pass temporal filtering $(0.01-0.1 \mathrm{~Hz})$ were conducted and then remove nuisance signal including white matter (WM) and cerebrospinal fluid (CSF), global mean signal regression, and 6 motion parameters. Finally, data quality control was performed and removed the participants whose maximal motion in each direction $>3 \mathrm{~mm}$, and rotation $>3^{\circ}$.

Data analysis. Calculating of network measures. After preprocessing of resting-state MRI data, the power 264 template was adopted to extract the time course of each ROI ${ }^{48}$. Then 10 well-defined brain networks (contain 227 nodes) were chosen to enter into the follow-up analysis, including the default mode network (DMN), salience network (SN), fronto-parietal network (FPN), cingulo-opercular network (CON), sensorimotor network (SMN), visual network (VN), ventral attention network (VAN), dorsal attention network (DAN), auditory network (AN) and subcortical network (SUB). The within-network connectivity was calculated as follows:

$$
W_{a}=\frac{\sum_{\mathrm{i}, \mathrm{j} \in \mathrm{a}} A_{i, j}}{N_{a}^{2}}
$$

For each brain network $(\mathrm{a} \in 1,2, \cdots, 10)$, the within-network connectivity was calculated as the average connectivity across all the links of the network normalized by the square of the number of nodes (ROIs). Aij represent the $227 \times 227$ connectivity matrix; $N a$ is the number of nodes within network $a ; i$ and $j$ notes the Power ROIs. Higher level of within-network connectivity indicated stronger interaction between those regions within the particular network.

The pairwise connectivity between networks was computed as follows: 


\begin{tabular}{|l|l|l|l|l|}
\hline & Network & r & p & PB H_FDR \\
\hline \multirow{4}{*}{ Within-network } & VAN & 0.144 & 0.001 & $<0.05$ \\
\cline { 2 - 5 } & DAN & 0.121 & 0.007 & $<0.05$ \\
\cline { 2 - 5 } & SN & 0.132 & 0.003 & $<0.05$ \\
\cline { 2 - 5 } Between-network & 0.146 & 0.001 & $<0.05$ \\
\hline & VAN-DAN & 0.172 & 0.001 & $<0.05$ \\
\cline { 2 - 5 } & VAN-SMN & 0.162 & 0.001 & $<0.05$ \\
\cline { 2 - 5 } & VAN-VN & 0.132 & 0.001 & $<0.05$ \\
\hline
\end{tabular}

Table 2. Within/between-network connectivity associated with ELS. Abbreviations: VAN, ventral attention network, DAN, dorsal attention network, SN, salience network, SMN, sensorimotor network, VN, visual network.

$$
P B_{a-b}=\frac{\sum_{\mathrm{i} \in \mathrm{a}, \mathrm{j} \in \mathrm{b}} A_{i, j}}{N_{a} N_{b}}
$$

The pairwise connectivity between networks $(P B a-b)$ was computed as the average connectivity across all the links between two networks, $a$ and $b(a, b \in 1,2, \cdots, 10 ; a \neq b)$, normalized by the product of the number of nodes within the two networks ${ }^{49}$. Higher level of between-network connectivity indicated stronger interaction between those regions of the two networks.

Brain-behavior correlation analyses. We conducted pearson correlations analysis between the within/ between-networks of connectivity and ELS, controlling for age and sex. The results were corrected by Benjamini and Hochberg FDR (BH_FDR) correction ${ }^{50}$.

Prediction analysis. To test the robustness of the brain-behavior relationship, we performed a machine-learning method named linear support vector regression (SVR) and cross-validation procedure with balanced ten-fold ${ }^{51}$. ELS was taken as the dependent variable and the network connectivity were taken as independent variables in the linear regression algorithm. The $r_{\text {(predicted, observed) }}$ was estimated by a four-fold cross-validation, and represent the prediction accuracy of the independent variable. We divided the data into four folds in order to keep the distributions of independent and dependent variables balanced. The prediction model was trained on the $75 \%$ sample and then was tested on the remaining participants. This procedure was conducted for 4 times to acquire a final $r_{\text {(predicted, observed) }}$ representing the correlation between the predict value and the actual value.

Generalization to a different sample. To examine to what extent the results could generalize to other sample, the same analysis was applied to Dataset 2. The analysis process was as follows: 1), preprocess the resting-state fMRI data, 2), construct brain networks based on the power 264 template, 3), calculate the within/between network connectivity, 4), Person correlation analysis was performed to investigate the relationship between ELS and network measures.

Mediation analysis. To examine whether the within-network connectivity of the VAN could explain the relationship between ELS and depression, a mediation analysis was conducted. The mediation model could partly explain the causal pathway by which the dependent variable $(\mathrm{Y})$ was affected by the independent variable (X) affects a dependent variable $(\mathrm{Y})$. Mediation analyses were performed by applying the indirect macro designed for SPSS $^{52}$. In the current study, $\mathrm{X}$ is the ELS, $\mathrm{Y}$ is the depression, and $\mathrm{M}$ is the within-network connectivity of the VAN. Age and sex were entered as covariates in the mediation model. This macro uses bootstrapped sampling to estimate the indirect mediation effect. In this alogorithm, 2,000 bootstrapped samples were drawn, and bias corrected $95 \%$ bootstrap confidence intervals (CI) were reported. CI that do not include zero indicate a significant indirect effect of the independent variable on the dependent variable through the mediators. Besides, the same mediating method was performed to explore whether attention bias of a sad face could explain the association between ELS and depression.

\section{Results}

The neural correlates of ELS. The demographic data are presented in Table 1. There was no gender difference in ELS $(t=2.682, p=0.377)$, and ELS was not related to age $(r=0.033, p=0.439)$. Within-network results indicated that ELS is positively correlated with within-network connectivity of the VAN, DAN, SMN-H, SN, and between-network of the VAN-DAN, VAN-VN, VAN-SMN (see Table 2 and Figure 1). Then prediction analysis was performed to test the robustness of the relationship between ELS and the within-network connectivity of these networks. Prediction results demonstrated that within/between-network connectivity of these networks could predict ELS ( $r=0.139, \mathrm{p}<0.005$, see Figure 2$)$, after controlling for age, gender and current stress. Due to the relatively small ample size of the Dataset 2 and its resultant low statistical power, the Dataset 2 only partly verified the present results. The results of Dataset 2 indicated that ELS was positively correlated with BDI score $(\mathrm{r}=0.230, \mathrm{p}<0.005)$, the within-network connectivity of the VAN $(\mathrm{r}=0.224, \mathrm{p}<0.005)$ and the DAN $(\mathrm{r}=0.165$, $\mathrm{p}<0.05)$. 


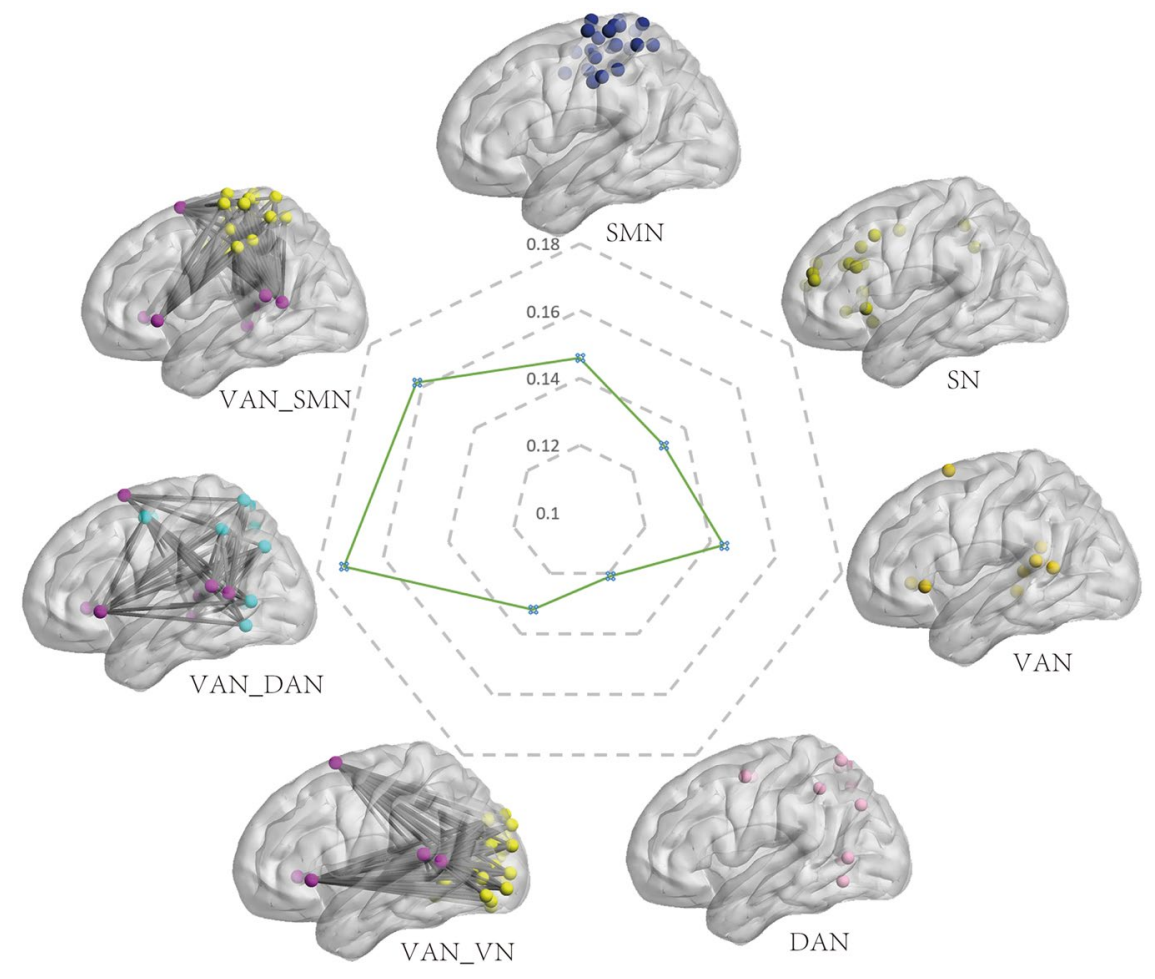

Figure 1. Within/between-network connectivity associated with ELS. The radar map shows the correlation coefficients of the within/between-network connectivity with ELS. Abbreviations: VAN, ventral attention network, DAN, dorsal attention network, SN, salience network, SMN, sensorimotor network, VN, visual network.

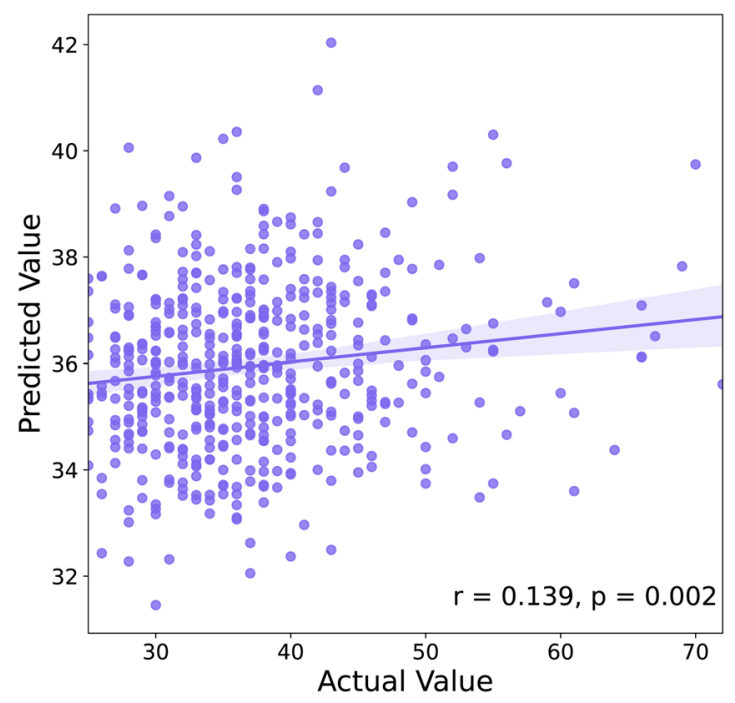

Figure 2. The prediction results. The within-network connectivity of VAN, DAN, SN, SMN and the betweennetwork connectivity of VAN_DAN, VAN_SN, VAN_SMN could predict individual difference of ELS.

Correlations between ELS, network measures and depression. A correlation analysis was conducted to explore the relationship between ELS, network measures and depression. After controlling for age, gender and current stress, ELS was significantly correlated with depression $(r=0.204, p<0.001)$, and the within-network connectivity of the VAN was also associated with depression $(r=0.137, p<0.005)$. These results suggested that a intensely association exists between the within-network connectivity of the VAN, ELS and depression. To test the hypothesis that attention bias sustains the relationship between ELS and depression, we also investigate the relationship between ELS, attention bias and depression. 


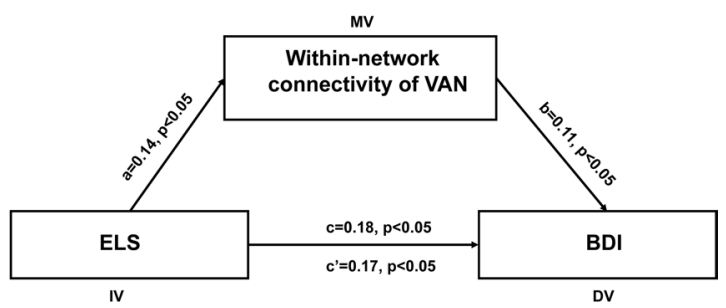

Figure 3. The within-network connectivity of VAN (Ventral attention network) mediate the relationship between ELS (Early life stress) and depression. The depicted diagram shows that ELS predicts displaced depression through the within-network connectivity of VAN after controlling for age, gender. Standardized regression coefficients are present in the path diagram.

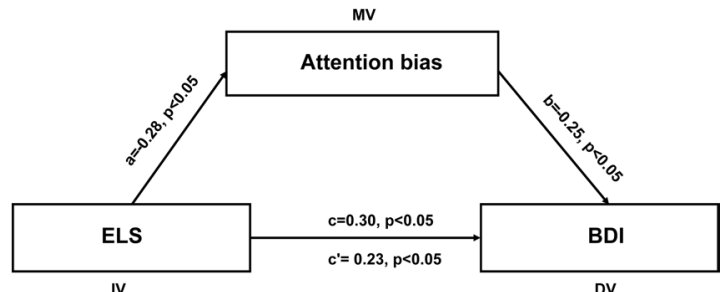

Figure 4. Attention bias mediate the relationship between ELS (Early life stress) and depression. The depicted diagram shows that ELS predicts displaced depression through attention bias after controlling for age, gender. Standardized regression coefficients are present in the path diagram.

Mediation results. The first mediation analysis indicated that the within-network connectivity of the VAN significantly mediate the relationship between ELS and depression (indirect effect $=0.01,95 \% \mathrm{CI}=[0.004,0.04]$, $\mathrm{p}<0.05$, see Figure 3), Standardized regression coefficients are present in the path diagram, which represent the covariant relationship between two variables. The second mediation analysis indicated that the attention bias to a sad face had a significant mediating effect on the relationship between ELS and depression (indirect effect $=0.07$, $95 \% \mathrm{CI}=[0.007,0.125], \mathrm{p}<0.05$, see Figure 4$)$

\section{Discussion}

This study extends existing knowledge about the neural basis of ELS by revealing that FC within/between large-scale brain networks were related to the individual differences of ELS. Fist, positive associations were observed between ELS and the within-network connectivity of the VAN, DAN, SN, SMN. Second, we found a positive relationship between ELS and the between-network connectivity of the VAN- DAN, VAN-VN, VAN-SMN. Finally, we found that attention bias and the within-network connectivity of the VAN mediate the relationship between ELS and depression. These results therefore provide a convincible neurobiological association for the changes in attention associated with childhood trauma and depression.

Positive associations were observed between ELS and the within-network connectivity of the VAN, DAN and the between-network connectivity of the VAN-DAN, which fitted well with previous studies that aberrant function of brain regions in attention network were associated with ELS and depression. For example, exposure to ELS was associated with increased activation of inferior parietal lobule during working memory task ${ }^{53}$. Previous studies detected increased functional connectivity between $\mathrm{dmpfc}$, dlpfc (key node of DAN), vmpfc and $\mathrm{ACC}^{54}$ in MDD patients. The VAN was confirmed to be involved in stimulus-driven attention ${ }^{29}$. For instance, detecting a salient target might require the involvement of the VAN (i.e., "invalid" targets in the Posner spatial cueing paradigm $)^{55-58}$. Whereas the DAN embodies top-down attention control ${ }^{59,60}$. DAN activity might increase when individuals orientate their attention to the current task ${ }^{56,61,62}$

Generally, these two networks tend to cooperate during normal cognitive processes. During an ongoing cognitive process, that task-relevant signals from the dorsal attention network might "filter" stimulus-driven signals in the ventral attention network, while stimulus-driven "circuit-breaking" signals from the ventral attention network offer an interruption to the dorsal attention network, reorienting the attention toward salient stimuli ${ }^{63}$. Several studies have demonstrated the association between childhood trauma and aberrant threat detection and response. Specifically, clinical study suggested that vigilance mediates the association between childhood neglect and negative symptoms in male psychotic patients ${ }^{64}$, exposure to ELS is associated with attention bias to a threatening stimulus, such as a fearful face $e^{65}$, or the emotional valence word ${ }^{66-68}$. Overall, this increased within/ between-network connectivity of attention systems might indicate a higher state of vigilance and awareness in individuals exposed to ELS.

In addition, the results also demonstrated that ELS was positively correlated with the within-network connectivity of the SN, SMN, and the between-network connectivity of VAN-VN, VAN-SMN. The SN is suggested to be involved in responding to behaviorally salient stimuli ${ }^{69}$ and implement the coordination of behavioral responses $^{70}$. Increased $\mathrm{SN}$ activity has been observed during situations in which changing behavior is more likely 
to be needed ${ }^{71}$. Previous study has indicated that the $\mathrm{SN}$ is involved in supporting stable attention for a current goal $^{72}$. In this study, this association between ELS and the within-network connectivity of the SN might suggest constant attention toward the external environment. The abnormal anatomical structure of the SMN is widely reported in ELS related studies ${ }^{73,74}$. The SMN is related to the processing of incoming sensory stimuli and generating motor outputs $\mathrm{s}^{75-79}$. Previous studies have suggested that depressive temperament was associated with alternated neuronal variability in the $\mathrm{SMN}^{80}$, Increased within-network connectivity of the SMN might support being more reactive to internal and external signals for initiating response. The VN was involved in processing primary visual information and projecting to higher sensory areas ${ }^{81}$. The stronger interaction between the SMN, VN and VAN might indicate a more sensitive perception of internal and external stimulus.

Further analysis revealed that there were positive association between the within-network connectivity of the VAN, attentional bias and ELS, which might suggest that adults who have experienced ELS have an altered within-network connectivity of the VAN associated with attention bias. In order to explore the neural and cognitive mechanisms for the influence of ELS on depression, we constructed two mediation models to decipher the potential relationship between ELS, depression, within-network connectivity of the VAN and attention bias. There is no doubt that attentional bias is a key cognitive feature in people with ELS, but the orientation of attentional bias is still controversial. Some studies indicated that ELS is related to attentional bias toward threatening or sad face ${ }^{82,83}$, while others suggested attentional bias away from threatening stimuli ${ }^{84,85}$. The present results supported the latter point. According to Posner's study, longer stimulus onset asynchronies (more than $300 \mathrm{~ms}$ ) might result in a reliable disadvantage for valid trails, during which the attention was first engaged by the cue and then disengaged from it (that is attention avoidance). Presumably, attention avoidance might be a defensive response to reducing exposure to negative stimuli, but from a long-term perspective it attentional avoidance might hinder effective emotion regulation ${ }^{86,87}$. Specifically, exposure to ELS might lead to attentional avoidance, which might associate with ineffective emotion regulation. Eventually, individual who has experienced ELS will develop a higher depressive state after stress events. Previous studies demonstrated that major depressive disorder was associated with abnormal connectivity of regions involved in cognitive control of attention ${ }^{88,89}$, indicating an aberrant attention process to salient cues. As for the role of the VAN in the relationship between ELS and depression, one possible explanation is that increased within-network connectivity of VAN might relate to a stronger stimulus-driven attention toward threaten stimuli, which conform to the sensibility to threatening stimuli in individual with higher depression.

Altogether, the present study provided behavioral and neural evidence for the alternations of ELS on individual's attention. Based on these results, we can infer that attention is a key factor to explain how individual who have experienced ELS develop depression. Besides, attention bias modification training could serve as a potential intervention method to reduce the negative impact of ELS.

Received: 14 January 2020; Accepted: 25 March 2020;

Published online: 09 April 2020

\section{References}

1. Teicher, M. H. \& Samson, J. A. Childhood maltreatment and psychopathology: A case for ecophenotypic variants as clinically and neurobiologically distinct subtypes. American journal of psychiatry 170, 1114-1133 (2013).

2. Thompson, M. P., Kaslow, N. J., Lane, D. B. \& Kingree, J. Childhood maltreatment, PTSD, and suicidal behavior among African American females. Journal of Interpersonal Violence 15, 3-15 (2000).

3. Grassi-Oliveira, R. \& Stein, L. M. Childhood maltreatment associated with PTSD and emotional distress in low-income adults: the burden of neglect. Child Abuse \& Neglect (2008).

4. Nemeroff, C. B. et al. Differential responses to psychotherapy versus pharmacotherapy in patients with chronic forms of major depression and childhood trauma. Proceedings of the National Academy of Sciences 100, 14293-14296 (2003).

5. Bernet, C. Z. \& Stein, M. B. Relationship of childhood maltreatment to the onset and course of major depression in adulthood. Depression and anxiety $9,169-174$ (1999).

6. Bifulco, A., Moran, P. M., Baines, R., Bunn, A. \& Stanford, K. Exploring psychological abuse in childhood: II. Association with other abuse and adult clinical depression. Bulletin of the Menninger Clinic 66, 241-258 (2002).

7. Simon, N. M. et al. Childhood maltreatment linked to greater symptom severity and poorer quality of life and function in social anxiety disorder. Depression and anxiety 26, 1027-1032 (2009).

8. Bruce, L. C., Heimberg, R. G., Blanco, C., Schneier, F. R. \& Liebowitz, M. R. Childhood maltreatment and social anxiety disorder: Implications for symptom severity and response to pharmacotherapy. Depression and Anxiety 29, 132-139 (2012).

9. Bar-Haim, Y., Lamy, D., Pergamin, L., Bakermans-Kranenburg, M. J. \& Van Ijzendoorn, M. H. Threat-related attentional bias in anxious and nonanxious individuals: a meta-analytic study. Psychological bulletin 133, 1 (2007).

10. Stein, J. A., Leslie, M. B. \& Nyamathi, A. Relative contributions of parent substance use and childhood maltreatment to chronic homelessness, depression, and substance abuse problems among homeless women: Mediating roles of self-esteem and abuse in adulthood. Child abuse \& neglect 26, 1011-1027 (2002).

11. Bellis, M. A., Hughes, K., Jones, A., Perkins, C. \& McHale, P. Childhood happiness and violence: a retrospective study of their impacts on adult well-being. BMJ open 3, e003427 (2013).

12. Nurius, P. S., Green, S., Logan-Greene, P. \& Borja, S. Life course pathways of adverse childhood experiences toward adult psychological well-being: A stress process analysis. Child abuse \& neglect 45, 143-153 (2015).

13. Romano, E., Babchishin, L., Marquis, R. \& Fréchette, S. Childhood maltreatment and educational outcomes. Trauma, Violence, \& Abuse 16, 418-437 (2015).

14. Pieterse, D. Childhood maltreatment and educational outcomes: Evidence from South Africa. Health economics 24, 876-894 (2015).

15. Corso, P. S., Edwards, V. J., Fang, X. \& Mercy, J. A. Health-related quality of life among adults who experienced maltreatment during childhood. American journal of public health 98, 1094-1100 (2008).

16. Morimoto, M., Morita, N., Ozawa, H., Yokoyama, K. \& Kawata, M. Distribution of glucocorticoid receptor immunoreactivity and mRNA in the rat brain: an immunohistochemical and in situ hybridization study. Neuroscience research 26, 235-269 (1996).

17. Caldji, C., Francis, D., Sharma, S., Plotsky, P. M. \& Meaney, M. J. The effects of early rearing environment on the development of GABAA and central benzodiazepine receptor levels and novelty-induced fearfulness in the rat. Neuropsychopharmacology 22, 219-229 (2000). 
18. Eiland, L., Ramroop, J., Hill, M. N., Manley, J. \& McEwen, B. S. Chronic juvenile stress produces corticolimbic dendritic architectural remodeling and modulates emotional behavior in male and female rats. Psychoneuroendocrinology 37, 39-47 (2012).

19. Cohen, M. M. et al. Early-life stress has persistent effects on amygdala function and development in mice and humans. Proceedings of the National Academy of Sciences 110, 18274-18278 (2013).

20. Teicher, M. H., Anderson, C. M. \& Polcari, A. Childhood maltreatment is associated with reduced volume in the hippocampal subfields CA3, dentate gyrus, and subiculum. Proceedings of the National Academy of Sciences 109, E563-E572 (2012).

21. Teicher, M. H. \& Samson, J. A. Annual research review: enduring neurobiological effects of childhood abuse and neglect. Journal of child psychology and psychiatry 57, 241-266 (2016).

22. Gosselin, L. \& Chia, F.-S. Characterizing temperate rocky shores from the perspective of an early juvenile snail: the main threats to survival of newly hatched Nucella emarginata. Marine Biology 122, 625-635 (1995).

23. Davis, J. S. et al. Attachment anxiety moderates the relationship between childhood maltreatment and attention bias for emotion in adults. Psychiatry research 217, 79-85 (2014).

24. Romens, S. E. \& Pollak, S. D. Emotion regulation predicts attention bias in maltreated children at-risk for depression. Journal of Child Psychology and Psychiatry 53, 120-127 (2012).

25. Gibb, B. E., Schofield, C. A. \& Coles, M. E. Reported history of childhood abuse and young adults' information-processing biases for facial displays of emotion. Child maltreatment 14, 148-156 (2009).

26. Bar-Haim, Y. et al. Life-threatening danger and suppression of attention bias to threat. American Journal of Psychiatry 167, 694-698 (2010).

27. Wald, I. et al. Battlefield-like stress following simulated combat and suppression of attention bias to threat. Psychological medicine 41, 699-707 (2011)

28. Sylvester, C. M. et al. Resting state functional connectivity of the ventral attention network in children with a history of depression or anxiety. Journal of the American Academy of Child \& Adolescent Psychiatry 52, 1326-1336. e1325 (2013).

29. Corbetta, M., Patel, G. \& Shulman, G. L. The reorienting system of the human brain: from environment to theory of mind. Neuron 58, 306-324 (2008)

30. Brown, J., Cohen, P., Johnson, J. G. \& Smailes, E. M. Childhood abuse and neglect: specificity of effects on adolescent and young adult depression and suicidality. Journal of the American Academy of Child \& Adolescent Psychiatry 38, 1490-1496 (1999).

31. Batten, S. V., Aslan, M., Maciejewski, P. K. \& Mazure, C. M. Childhood maltreatment as a risk factor for adult cardiovascular disease and depression. Journal of Clinical Psychiatry 65, 249-254 (2004).

32. Raes, F. \& Hermans, D. On the mediating role of subtypes of rumination in the relationship between childhood emotional abuse and depressed mood: Brooding versus reflection. Depression and Anxiety 25, 1067-1070 (2008).

33. Gibb, B., Alloy, L. \& Abramson, L. Global reports of childhood maltreatment versus recall of specific maltreatment experiences: Relationships with dysfunctional attitudes and depressive symptoms. Cognition and Emotion 17, 903-915 (2003).

34. MacLeod, C., Rutherford, E., Campbell, L., Ebsworthy, G. \& Holker, L. Selective attention and emotional vulnerability: assessing the causal basis of their association through the experimental manipulation of attentional bias. Journal of abnormal psychology 111, 107 (2002).

35. Suzuki, H. et al. Early Life Stress and Trauma and Enhanced Limbic Activation to Emotionally Valenced Faces in Depressed and Healthy Children. Journal of the American Academy of Child \& Adolescent Psychiatry 53, 800-813.e810.

36. Hakamata, Y. et al. Attention bias modification treatment: a meta-analysis toward the establishment of novel treatment for anxiety. Biological psychiatry 68, 982-990 (2010).

37. Peckham, A. D., McHugh, R. K. \& Otto, M. W. A meta-analysis of the magnitude of biased attention in depression. Depression and anxiety 27, 1135-1142 (2010).

38. Foa, E. B. \& Kozak, M. J. Emotional processing of fear: exposure to corrective information. Psychological bulletin 99, 20 (1986).

39. Clark, D. M. \& Wells, A. A cognitive model of social phobia. Social phobia: Diagnosis, assessment, and treatment 41, 00022-00023 (1995).

40. Yang, W., Ding, Z., Dai, T., Peng, F. \& Zhang, J. X. Attention bias modification training in individuals with depressive symptoms: a randomized controlled trial. Journal of behavior therapy and experimental psychiatry 49, 101-111 (2015).

41. Bernstein, D. P. et al. Development and validation of a brief screening version of the Childhood Trauma Questionnaire. Child abuse \& neglect 27, 169-190 (2003).

42. Fink, L. A., Bernstein, D., Handelsman, L., Foote, J. \& Lovejoy, M. Initial reliability and validity of the childhood trauma interview: a new multidimensional measure of childhood interpersonal trauma. The American journal of psychiatry (1995).

43. Beck, A. T., Steer, R. A. \& Brown, G. K. Beck depression inventory-II. San Antonio 78, 490-498 (1996).

44. Beck, A. T., Steer, R. A. \& Carbin, M. G. Psychometric properties of the Beck Depression Inventory: Twenty-five years of evaluation. Clinical psychology review 8, 77-100 (1988).

45. Liu, X., Liu, L., Yang, J. \& Zhao, G. Reliability and validity of the adolescents self-rating life events checklist. Chinese Journal of Clinical Psychology 5, 34-36 (1997).

46. MacLeod, C., Mathews, A. \& Tata, P. Attentional bias in emotional disorders. Journal of abnormal psychology 95, 15 (1986).

47. Gotlib, I. H., Krasnoperova, E., Yue, D. N. \& Joormann, J. Attentional biases for negative interpersonal stimuli in clinical depression. Journal of abnormal psychology 113, 127 (2004).

48. Power, J. D. et al. Functional network organization of the human brain. 72, 665-678 (2011).

49. Yu, M. et al. Childhood trauma history is linked to abnormal brain connectivity in major depression. Proceedings of the National Academy of Sciences 116, 8582-8590 (2019).

50. Benjamini, Y. \& Hochberg, Y. Controlling the false discovery rate: a practical and powerful approach to multiple testing. Journal of the Royal statistical society: series B (Methodological) 57, 289-300 (1995).

51. Supekar, K. et al. Neural predictors of individual differences in response to math tutoring in primary-grade school children. Proceedings of the National Academy of Sciences 110, 8230-8235 (2013).

52. Preacher, K. J. \& Hayes, A. F. Asymptotic and resampling strategies for assessing and comparing indirect effects in multiple mediator models. Behavior research methods 40, 879-891 (2008).

53. Quidé, Y. et al. Effects of childhood trauma on working memory in affective and non-affective psychotic disorders. Brain imaging and behavior 11, 722-735 (2017).

54. Sheline, Y. I. et al. Increased amygdala response to masked emotional faces in depressed subjects resolves with antidepressant treatment: an fMRI study. Biological psychiatry 50, 651-658 (2001).

55. Arrington, C. M., Carr, T. H., Mayer, A. R. \& Rao, S. M. Neural mechanisms of visual attention: object-based selection of a region in space. Journal of cognitive neuroscience 12, 106-117 (2000).

56. Corbetta, M., Kincade, J. M., Ollinger, J. M., McAvoy, M. P. \& Shulman, G. L. Voluntary orienting is dissociated from target detection in human posterior parietal cortex. Nature neuroscience 3, 292 (2000).

57. Kincade, J. M., Abrams, R. A., Astafiev, S. V., Shulman, G. L. \& Corbetta, M. An event-related functional magnetic resonance imaging study of voluntary and stimulus-driven orienting of attention. Journal of Neuroscience 25, 4593-4604 (2005).

58. Macaluso, E., George, N., Dolan, R., Spence, C. \& Driver, J. Spatial and temporal factors during processing of audiovisual speech: a PET study. Neuroimage 21, 725-732 (2004).

59. Bundesen, C. Visual selection of features and objects: Is location special? A reinterpretation of Nissen's (1985) findings. Perception \& Psychophysics 50, 87-89 (1991). 
60. Desimone, R. \& Duncan, J. Neural mechanisms of selective visual attention. Annual review of neuroscience 18, 193-222 (1995).

61. Hopfinger, J. B., Buonocore, M. H. \& Mangun, G. R. The neural mechanisms of top-down attentional control. Nature neuroscience 3, $284(2000)$.

62. Pessoa, L., Kastner, S. \& Ungerleider, L. G. Neuroimaging studies of attention: from modulation of sensory processing to top-down control. Journal of Neuroscience 23, 3990-3998 (2003).

63. Corbetta, M. \& Shulman, G. L. Control of goal-directed and stimulus-driven attention in the brain. Nature reviews neuroscience 3 , 201 (2002).

64. Mansueto, G. et al. Childhood adversities and psychotic symptoms: the potential mediating or moderating role of neurocognition and social cognition. Schizophrenia research 206, 183-193 (2019).

65. Choi, K., Jeon, G.-S. \& Cho, S.-I. Prospective study on the impact of fear of falling on functional decline among community dwelling elderly women. International journal of environmental research and public health 14, 469 (2017).

66. Wingenfeld, K. et al. Associations of childhood trauma, trauma in adulthood and previous-year stress with psychopathology in patients with major depression and borderline personality disorder. Child Abuse \& Neglect: The International Journal 35, 647-654 (2011).

67. McNally, R. J., Clancy, S. A. \& Schacter, D. L. Directed forgetting of trauma cues in adults reporting repressed or recovered memories of childhood sexual abuse. Journal of abnormal psychology 110, 151 (2001).

68. Puetz, V. B. et al. Altered neural response to rejection-related words in children exposed to maltreatment. Journal of child psychology and psychiatry 57, 1165-1173 (2016).

69. Seeley, W. W., Crawford, R. K., Zhou, J., Miller, B. L. \& Greicius, M. D. Neurodegenerative diseases target large-scale human brain networks. Neuron 62, 42-52 (2009).

70. Medford, N. \& Critchley, H. D. Conjoint activity of anterior insular and anterior cingulate cortex: awareness and response. Brain Structure and Function 214, 535-549 (2010).

71. Dosenbach, N. U. et al. Distinct brain networks for adaptive and stable task control in humans. Proceedings of the National Academy of Sciences 104, 11073-11078 (2007).

72. Beaty, R. E., Benedek, M., Kaufman, S. B. \& Silvia, P. J. Default and executive network coupling supports creative idea production. Scientific reports 5, 10964 (2015).

73. Heim, C. M., Mayberg, H. S., Mletzko, T., Nemeroff, C. B. \& Pruessner, J. C. Decreased cortical representation of genital somatosensory field after childhood sexual abuse. American Journal of Psychiatry 170, 616-623 (2013).

74. Khan, A. et al. Childhood maltreatment, depression, and suicidal ideation: critical importance of parental and peer emotional abuse during developmental sensitive periods in males and females. Frontiers in psychiatry 6, 42 (2015).

75. Basalyga, G. \& Salinas, E. When response variability increases neural network robustness to synaptic noise. Neural Computation 18, $1349-1379$ (2006).

76. Faisal, A. A., Selen, L. P. \& Wolpert, D. M. Noise in the nervous system. Nature reviews neuroscience 9, 292 (2008).

77. Garrett, D. D., McIntosh, A. R. \& Grady, C. L. Moment-to-moment signal variability in the human brain can inform models of stochastic facilitation now. Nature Reviews Neuroscience 12, 612 (2011).

78. Zou, Q.-H. et al. An improved approach to detection of amplitude of low-frequency fluctuation (ALFF) for resting-state fMRI: fractional ALFF. Journal of neuroscience methods 172, 137-141 (2008).

79. Zuo, X.-N. et al. The oscillating brain: complex and reliable. Neuroimage 49, 1432-1445 (2010).

80. Conio, B. et al. Opposing patterns of neuronal variability in the sensorimotor network mediate cyclothymic and depressive temperaments. Human brain mapping 40, 1344-1352 (2019).

81. Thomas Yeo, B. et al. The organization of the human cerebral cortex estimated by intrinsic functional connectivity. Journal of neurophysiology 106, 1125-1165 (2011).

82. Fani, N. et al. Attention bias toward threat is associated with exaggerated fear expression and impaired extinction in PTSD. Psychological medicine 42, 533-543 (2012).

83. Gibb, B. E., Benas, J. S., Grassia, M. \& McGeary, J. Children's attentional biases and 5-HTTLPR genotype: Potential mechanisms linking mother and child depression. Journal of Clinical Child \& Adolescent Psychology 38, 415-426.

84. Mansell, W. \& Clark, D. M. How do I appear to others? Social anxiety and processing of the observable self. Behaviour research and therapy 37, 419-434 (1999).

85. Pishyar, R., Harris, L. M. \& Menzies, R. G. Attentional bias for words and faces in social anxiety. Anxiety, Stress \& Coping 17, 23-36 (2004).

86. Koole, S. L. The psychology of emotion regulation: An integrative review. Cognition and emotion 23, 4-41 (2009).

87. Werner, K. H., Goldin, P. R., Ball, T. M., Heimberg, R. G. \& Gross, J. J. Assessing emotion regulation in social anxiety disorder: The emotion regulation interview. Journal of Psychopathology and Behavioral Assessment 33, 346-354 (2011).

88. Kaiser, R. H., Andrews-Hanna, J. R., Wager, T. D. \& Pizzagalli, D. A. Large-scale network dysfunction in major depressive disorder: a meta-analysis of resting-state functional connectivity. JAMA psychiatry 72, 603-611 (2015).

89. Kaiser, R. H. et al. Distracted and down: neural mechanisms of affective interference in subclinical depression. Social cognitive and affective neuroscience 10, 654-663 (2014).

\section{Acknowledgements}

This research was supported by the Chongqing doctoral research and innovation project (CYB19102). National Natural Science Foundation of China (31470981; 31571137; 31500885; 31600878; 31771231), Project of the National Defense Science and Technology Innovation Special Zone, Chang Jiang Scholars Program, National Outstanding Young People Plan, the Program for the Top Young Talents by Chongqing, the Fundamental Research Funds for the Central Universities (SWU1609177), Natural Science Foundation of Chongqing (cstc2015jcyjA10106), Fok Ying Tung Education Foundation (151023), the Research Program Funds of the Collaborative Innovation Center of Assessment toward Basic Education Quality at Beijing Normal University.

\section{Author contributions}

J.Q. and C.D. initiated and supervised this project. J.Q. and Y.M. conceived and designed the experiment. Y.M. and H.X. interpreted the results and write the manuscript. All authors contributed to the manuscript.

\section{Competing interests}

The authors declare no competing interests.

\section{Additional information}

Correspondence and requests for materials should be addressed to C.D. or J.Q.

Reprints and permissions information is available at www.nature.com/reprints. 
Publisher's note Springer Nature remains neutral with regard to jurisdictional claims in published maps and institutional affiliations.

(c) (i) Open Access This article is licensed under a Creative Commons Attribution 4.0 International License, which permits use, sharing, adaptation, distribution and reproduction in any medium or format, as long as you give appropriate credit to the original author(s) and the source, provide a link to the Creative Commons license, and indicate if changes were made. The images or other third party material in this article are included in the article's Creative Commons license, unless indicated otherwise in a credit line to the material. If material is not included in the article's Creative Commons license and your intended use is not permitted by statutory regulation or exceeds the permitted use, you will need to obtain permission directly from the copyright holder. To view a copy of this license, visit http://creativecommons.org/licenses/by/4.0/.

(c) The Author(s) 2020 